\title{
Serum Apolipoprotein A-I Predicts Response of Rectal Cancer to Neoadjuvant Chemoradiotherapy
}

This article was published in the following Dove Press journal: Cancer Management and Research

\author{
Su-ping Guo ${ }^{1,2, *}$ \\ Chen Chen ${ }^{1,2, *}$ \\ Zhi-fan Zeng (iD) ${ }^{1,2}$ \\ Qiao-xuan Wang ${ }^{1,2}$ \\ Wu Jiang ${ }^{2,3}$ \\ Yuan-hong Gao (iD) ${ }^{1,2}$ \\ Hui Chang (D) ${ }^{1,2}$ \\ 'Department of Radiation Oncology, Sun \\ Yat-sen University Cancer Center, \\ Guangzhou, People's Republic of China; \\ ${ }^{2}$ State Key Laboratory of Oncology in \\ South China, Collaborative Innovation \\ Center for Cancer Medicine, Guangzhou, \\ People's Republic of China; ${ }^{3}$ Department \\ of Colorectal Surgery, Sun Yat-sen \\ University Cancer Center, Guangzhou, \\ People's Republic of China
}

*These authors contributed equally to this work
Correspondence: Yuan-hong Gao; Hui Chang

Department of Radiation Oncology, Sun Yat-sen University Cancer Center, 65I

Dongfeng Road East, Guangzhou,

Guangdong, 510060, People's Republic of China

Tel +86-I3560I82I68; +86-I3480295989

Email gaoyh@sysucc.org.cn;

changhui@sysucc.org.cn
Background: Serum lipids have been reported as prognosticators for malignancies, including rectal cancer $(\mathrm{RC})$. Yet, their value in predicting the response of $\mathrm{RC}$ to neoadjuvant chemoradiotherapy (NACRT) remains unknown. This study aimed to assess the predictive abilities of serum lipids for a bad response, and to build a serum lipid-based prediction model.

Methods: In total, 751 patients diagnosed with stage cII-III RC and treated with NACRT plus surgery from January 2007 to August 2018 were retrospectively reviewed and randomly divided into two data sets, in a ratio of 1:1. Receiver operating characteristics (ROC) analysis was conducted in the development set to select possible predictors of bad NACRT response from pathoclinical factors, including serum lipids. Multivariate logistic regression was conducted to further determine independent predictors, which were then used to develop a prediction index (PI). Finally, the PI was verified in the validation set, through ROC analysis and chi-squared test.

Results: Five independent predictors were identified: tumor length $\geq 4 \mathrm{~cm}$, cT4 stage, carcinoembryonic antigen $\geq 5.0 \mathrm{ng} / \mathrm{mL}$, irradiation with three-dimensional conformal radiotherapy technique, and apolipoprotein $\mathrm{A}-\mathrm{I} \leq 1.20 \mathrm{~g} / \mathrm{L}$. Each of them was assigned a number of points. In the validation set, the area under the curve of PI appeared as 0.642 (95\% confidence interval $0.586-0.697$ ). The sensitivity, specificity, positive and negative predictive values, and concordance were $72.3 \%, 52.3 \%, 63.8 \%, 61.9 \%$, and $63.0 \%$, respectively.

Conclusion: Serum apolipoprotein A-I was found to correlate negatively with the RC response to NACRT. It could serve as a biomarker for guiding individualized treatment and a potential target for improving sensitivity to chemoradiation.

Keywords: rectal cancer, radiotherapy, apolipoprotein A-I, tumor response, prediction model

\section{Introduction}

Neoadjuvant chemoradiotherapy (NACRT) followed by surgery is now the standard management of locally advanced rectal cancer (RC), owing to its superiority in improving local control and the likelihood of sphincter preservation. ${ }^{1}$ Furthermore, it is generally accepted that tumor response to NACRT is independently associated with patients' long-term survival. However, an ideal response is seen in only $14.5-31.2 \%$ of RC patients. ${ }^{2,3}$ Tumor response may be improved by increasing the intensity of NACRT, such as with an irradiation boost through brachytherapy. Simultaneously, this boost can also lead to an increased risk of severe toxicity, such as acute and late proctitis. ${ }^{4}$ Hence, it is important to identify those patients who harbor NACRT-resistant tumors and really need intensified treatment. 
Pretreatment anatomical, genetic, and immunological features of $\mathrm{RC}$ have been proven to predict its response to NACRT. $^{5-7}$ On the other side, serum indices of patients could also act as predictors of NACRT response. A series of studies has confirmed the predictive ability of serum carcinoembryonic antigen (CEA) in pathological complete response (pCR) ${ }^{8-10}$ In a study by Song et al, carbohydrate antigen 19-9 (CA19-9) appeared to predict downstaging to stage $0-\mathrm{I} .{ }^{11}$ Even indices from the complete blood count, such as the platelet-lymphocyte ratio, have been shown to predict $\mathrm{pCR}^{12}$ These indices provide non-invasive, economic, and practical choices for response prediction.

Serum lipids are a group of indices which can be easily tested, and established intervention methods are available for some of them. ${ }^{13}$ In RC, dyslipidemia was reported to be associated with an increased risk of oncogenesis and progression. ${ }^{14,15}$ A study by Del Boccio et al correlated levels of some serum lipids, including sphingomyelin, lysophosphatidylcholine, and lysophosphatidylethanolamine, with NACRT response, ${ }^{16}$ but the small sample size and seldomly used indices limit the clinical application of its results. Here, we involved a larger cohort to evaluate the value of routinely tested serum lipids in predicting resistant $\mathrm{RC}$, and tried to develop a prediction model based on serum lipids.

\section{Patients and Methods}

\section{Study Subjects}

The inclusion criteria for this study were: 1) age between 18 and 75 years; 2) RC initially diagnosed in our hospital from January 1st 2007 to August 31st 2018; 3) pretherapeutic clinical stage II-III (cT3-4N0M0, cT1-4N12M0); and 4) complete records of NACRT, radical (R0) resection and postsurgical pathological examinations. The exclusion criteria were: 1) Karnofsky performance score $<70$; 2) any severe cardiac, pulmonary, hepatic, renal, or hematopoietic comorbidities unsuitable for chemotherapy or radiotherapy; 3 ) any other prior malignancies; 4) history of pelvic irradiation; 5) distant metastasis before or during radiotherapy; 6) application of monoclonal antibody; and/ or 7) regular use of lipid-modulating drugs, such as niacins and statins, before treatment. The clinical data of the eligible patients were retrospectively reviewed.

\section{Diagnosis, Staging, and Tumor Response Evaluation}

Before treatment, biopsy under rectoscope was performed to obtain a pathological diagnosis. Local invasion and lymph- node metastasis were detected by magnetic resonance imaging of the pelvis and endoscopic ultrasonography. A whole-body bone scan and computed tomography of the chest and abdomen were performed to detect metastasis in distant organs, such as bone, lung, and liver. Suspicious distant metastases were confirmed by positron emission tomography. Serum levels of CEA and CA19-9 were tested before treatment, using an E170 electrochemiluminescent immunoassay system (Roche Diagnostics, Tokyo, Japan).

After surgery, pathological examinations of resected specimens were performed by two pathologists specialized in gastrointestinal cancers, including quality of total mesorectal excision (TME), tumor infiltration and differentiation, numbers of examined and involved lymph nodes, and tumor regression grade (TRG).

Both pretreatment clinical staging and postsurgical pathological staging were based on the TNM classification of the Union for International Cancer Control-American Joint Cancer Committee. TRG was based on the Mandard's five-tier grading system, ${ }^{3}$ in which TRG 5-3 and $2-1$ were considered as bad and good responses, respectively.

\section{Serum Lipid Assessment}

A LABOSPECT 008 biochemistry system (Hitachi, Tokyo, Japan) was used to assess serum lipids before treatment, including total cholesterol (TC), triglyceride (TG), high-density lipoprotein cholesterol (HDL-c), lowdensity lipoprotein cholesterol (LDL-c), apolipoprotein A-I (apoAI), and apolipoprotein B (apoB).

\section{Neoadjuvant Treatment and Surgery}

Target delineation of three-dimensional conformal and intensified modulated radiation therapy (3DCRT and IMRT) was based on the guidelines of the International Commission on Radiation Units and Measurements Reports 50 and 62. Then, a linear accelerator delivering an 8-MV photon beam was used to perform irradiation, in a conventional fractionation (2 Gy per fraction, one fraction per day, 5 days per week). Macroscopic tumor (including primary lesion and enlarged lymph nodes) and high-risk (including pararectal, presacral, obturator, internal, and common iliac) lymphatic drainage regions were irradiated to total doses of 50 and $46 \mathrm{~Gy}$, respectively.

Neoadjuvant chemotherapy was administered in two modes: 1) a total of two cycles during radiotherapy; or 2) a total of four cycles, comprising one cycle before, two cycles during, and one cycle after radiotherapy. The 
regimen was capecitabine plus oxaliplatin (CAPEOX) with a 21-day cycle. In each cycle, capecitabine was given $1000 \mathrm{mg} / \mathrm{m}^{2}$ twice daily, on days $1-14$, and oxaliplatin was given $130 \mathrm{mg} / \mathrm{m}^{2}$ on day 1 .

Radical surgery was scheduled 6-8 weeks after the last cycle of neoadjuvant chemotherapy, according to the standard of TME. Completeness of TME was ensured by intraoperative pathological examination on frozen sections of resection margin.

\section{Data Set Definition}

The eligible patients were divided into two data sets in a ratio of 1:1, using the 'Select Cases: Random sample of cases' function of IBM SPSS Statistics 23.0 (IBM Corp., Armonk, NY, USA). One data set was called the development set because it was used to evaluate correlations between serum lipid level and bad response, and to develop a serum lipidbased prediction model. The other data set was called the validation set because it was used to validate the predictive efficiency and accuracy of the model originating from the development set. The balance of baseline pathoclinical features between the development and validation sets was tested using Mann-Whitney U and chi-squared tests for continuous and categorical data, respectively.

\section{Variables and Cut-off Values}

The candidate variables to develop the prediction model included age, gender (female vs male), tumor differentiation (high vs moderate-low), tumor length, clinical $\mathrm{T}$ (cT4 vs cT3-1) and $\mathrm{N}$ (cN+ vs cN0) stages, anemia (yes vs no), CEA, CA19-9, irradiation technique (3DCRT vs IMRT), number of chemotherapy cycles (2 vs 4), TC, TG, HDL-c, LDL-c, apoAI, and apoB. For convenience in use, the continuous variables were all altered to a binomial form. The cut-off value of age was its median value in the development set. The cutoff value of tumor length was $4.0 \mathrm{~cm}$, which referred to the study by $\mathrm{Hu}$ et al. $^{9}$ The cut-off values of CEA, CA19-9, TC, TG, HDL-c, LDL-c, apoAI, and apoB were $5.0 \mathrm{ng} / \mathrm{mL}, 35.0 \mathrm{U} / \mathrm{mL}, 5.69 \mathrm{mmol} / \mathrm{L}, 1.70 \mathrm{mmol} /$ $\mathrm{L}, 1.29 \mathrm{mmol} / \mathrm{L}, 3.10 \mathrm{mmol} / \mathrm{L}, 1.20 \mathrm{~g} / \mathrm{L}$, and $1.10 \mathrm{~g} / \mathrm{L}$, respectively, as recommended by the manufacturers of the assay kits.

\section{Model Development}

First, the abilities of each variable to predict a bad response were tested by a receiver operating characteristics (ROC) analysis and confirmed by a chi-squared test. The variables exhibiting predictive abilities in the univariate analyses were entered into a multivariate logistic regression. Second, a backward selection (likelihood ratio) method was used to select the variables with prediction independence for the prediction model. The odds ratio (OR), 95\% confidence interval (CI), and $\beta$ regression coefficient were calculated for each independent predictor, which was then assigned with an integer point nearest to its $\beta$ coefficient divided by the smallest $\beta$ coefficient. Third, the prediction index (PI) of a patient was obtained by summing the points of all the variables. The best cut-off value of PI was determined through ROC analysis and based on its Youden index.

\section{Model Validation and Calibration}

The cut-off value of the PI was first validated in the development set, through a chi-squared test. Then, the PIs of all the patients in the validation set were calculated. The predictive efficiency of the PI was measured on the basis of its area under the curve (AUC) in ROC analysis. To measure the predictive accuracy of the PI, the sensitivity, specificity, positive and negative predictive values, and concordance of its cut-off value were calculated, also through a chi-squared test. Finally, the goodness-of-fit of the PI was evaluated through a Hosmer-Lemeshow test, in both the development and validation sets.

All of the statistical analyses in this study were performed using IBM SPSS Statistics 23.0 and GraphPad Prism 6.0 (GraphPad Software, San Diego, CA, USA). A difference with a two-sided $P$ value of $<0.05$ was considered to be statistically significant. The whole procedure of this study is summarized in Figure 1.

\section{Ethics Approval and Consent to Participate}

All patients signed informed consent before treatment. This study was approved by the Institutional Review Board of the Sun Yat-sen University Cancer Center (approval no. B2020-384-01). This study was conducted in accordance with the Declaration of Helsinki.

\section{Results}

\section{Enrollment and Grouping}

In total, 751 patients were diagnosed with stage cII-III RC, managed with NACRT plus surgery, and eligible for this study. After randomized division, there were 375 and 376 cases in the development and validation sets, respectively. No differences were seen between these two data sets in age, 


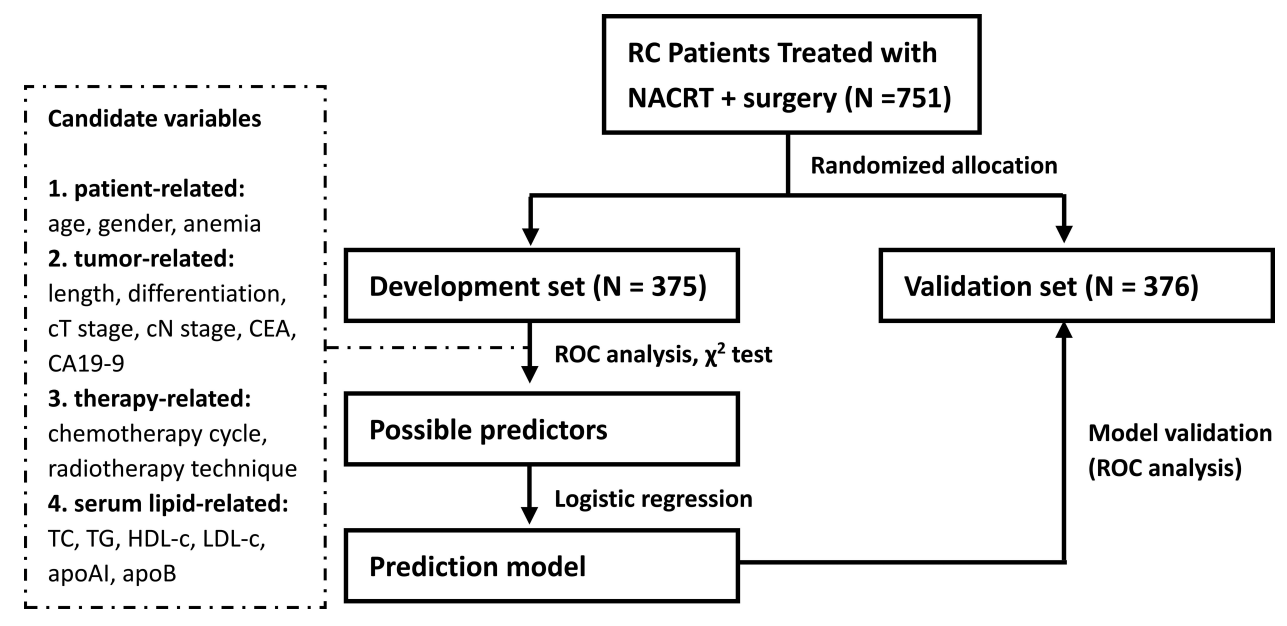

Figure I Procedure of this study.

Abbreviations: RC, rectal cancer; NACRT, neoadjuvant chemoradiotherapy; CEA, carcinoembryonic antigen; CAI9-9, carbohydrate antigen 19-9; TC, total cholesterol, TG, triglyceride; HDL-c, high-density lipoprotein cholesterol; LDL-c, low-density lipoprotein cholesterol; apoAl, apolipoprotein A-l; apoB, apolipoprotein B; ROC, receiver operating characteristics.

gender, tumor differentiation, tumor length, clinical $\mathrm{T}$ or $\mathrm{N}$ stage, hemoglobin, CEA, CA19-9, irradiation technique, or chemotherapy cycle (Table 1). That is, the two sets had balanced baseline pathoclinical characteristics, which made it feasible to validate the model in the validation set. In addition, the median age in the development set was 57 (range 22-75) years old. This figure was also used as the cutoff value for age in the following analyses.

\section{Model Development}

In ROC analyses, tumor length, clinical $\mathrm{T}$ stage, CEA, irradiation technique, and apoAI emerged as possible predictors of bad response (Figure 2). Their AUCs were $0.595,0.561,0.609,0.563$, and 0.415 ( $P$ values were $0.001,0.040,<0.001,0.034$, and 0.005$)$, respectively. When altered to a binomial form (Figure S1), the AUCs for tumor length ( $\geq 4.0 \mathrm{vs}<4.0 \mathrm{~cm}$ ), CEA ( $\geq 5.0 \mathrm{vs}<5.0 \mathrm{ng} /$ $\mathrm{mL})$, and apoAI $(\leq 1.20 \mathrm{vs}>1.20 \mathrm{~g} / \mathrm{L})$ were $0.578,0.567$, and 0.577 ( $P$ values were $0.009,0.024$, and 0.010$)$, respectively. Their predictive abilities were further confirmed by the chi-squared test (Table 2). Other serum lipid indices, including TC, TG, HDL-c, LDL-c, and apoB, all failed to predict a bad response (Table S1).

Next, tumor length $\geq 4.0 \mathrm{~cm}$, cT4 stage, CEA $\geq 5.0$ $\mathrm{ng} / \mathrm{mL}, 3 \mathrm{DCRT}$, and apoAI $\leq 1.20 \mathrm{~g} / \mathrm{L}$ were entered into a multivariate logistic regression to test their independence in predicting a bad response and building a prediction model (Table 3). As a result, all five of these variables remained as independent predictors $(P$ values were $0.007,0.033,0.038,0.042$, and 0.038 , respectively) and were assigned with points. After summing the points for each patient, the PI in the development set ranged 0 to 5 (median=2). Through ROC analysis, the best cut-off value was calculated to be 2 $(\geq 2$ vs $<2$ ), with a maximum Youden index of 0.248 (Figure 3A).

\section{Model Validation and Calibration}

In the development set, the chi-squared test showed that PI $\geq 2$ was associated with a higher proportion of bad responders, compared with PI $<2(61.7 \%$ vs $34.8 \%, P<0.001)$ (Figure 3B). The PI was also calculated for each patient in the validation set. Its AUC in ROC analysis appeared to be 0.642 (95\% CI 0.586-0.697) (Figure 3C). A higher proportion of bad responders was also observed in the cases with PI $\geq 2$, compared with those with PI $<2(63.8 \%$ vs $38.1 \%, P<0.001$ ) (Figure 3D). In other words, the predictive ability of $\mathrm{PI}=2$ was confirmed in the validation set. The sensitivity, specificity, positive and negative predictive values, and concordance of our model were $72.3 \%, 52.3 \%$, $63.8 \%, 61.9 \%$, and $63.0 \%$, respectively.

In Hosmer-Lemeshow goodness-of-fit tests on the PI, the chi-square, degrees of freedom, and $P$ value of the development set were $1.113,3$, and 0.774 , respectively. The corresponding figures for the validation set were $3.785,3$, and 0.286 , respectively. This indicated that the PI did not deviate significantly from a perfect fit in either of the two data sets. The calibration curves of the PI are shown in Figure S2. 
Table I Baseline Pathoclinical Features in the Development and Validation Sets

\begin{tabular}{|c|c|c|c|}
\hline Features & $\begin{array}{l}\text { Development } \\
\text { Set }(N=375)\end{array}$ & $\begin{array}{l}\text { Validation Set } \\
(\mathbf{N}=376)\end{array}$ & $P$ value \\
\hline Age (years) & $57(22-75)$ & $56(18-75)$ & 0.370 \\
\hline $\begin{array}{c}\text { Gender } \\
\text { Female } \\
\text { Male }\end{array}$ & $\begin{array}{l}132(35.2 \%) \\
243(64.8 \%)\end{array}$ & $\begin{array}{l}129(36.0 \%) \\
247(64.0 \%)\end{array}$ & 0.815 \\
\hline $\begin{array}{l}\text { Tumor } \\
\text { differentiation } \\
\text { High } \\
\text { Moderate- } \\
\text { low }\end{array}$ & $\begin{array}{l}4 \mathrm{I}(5 \mathrm{I} .9 \%) \\
334(49.7 \%)\end{array}$ & $\begin{array}{l}38(48.1 \%) \\
338(50.3 \%)\end{array}$ & 0.712 \\
\hline $\begin{array}{l}\text { Tumor length } \\
(\mathrm{cm})\end{array}$ & $3.2(1.0-15.0)$ & $3.0(1.0-12.0)$ & 0.683 \\
\hline $\begin{array}{l}\text { Clinical T stage } \\
\text { cT4 } \\
\text { cT3-I }\end{array}$ & $\begin{array}{l}\text { I5I (40.3\%) } \\
224(59.7 \%)\end{array}$ & $\begin{array}{l}167(44.4 \%) \\
209(55.6 \%)\end{array}$ & 0.250 \\
\hline $\begin{array}{l}\text { Clinical N stage } \\
\mathrm{cN}+ \\
\mathrm{cN} 0\end{array}$ & $\begin{array}{l}306(81.6 \%) \\
69(18.4 \%)\end{array}$ & $\begin{array}{l}311(82.7 \%) \\
65(17.3 \%)\end{array}$ & 0.690 \\
\hline $\begin{array}{l}\text { Hemoglobin (g/ } \\
\text { L) } \\
\text { CEA }(\mathrm{ng} / \mathrm{mL}) \\
\text { CAI9-9 (U/mL) }\end{array}$ & $\begin{array}{l}132(67-174) \\
4.0(0.2-394.0) \\
13.6(0.0-458.0)\end{array}$ & $\begin{array}{l}\text { I34 (7|-173) } \\
4.4(0.0-480.8) \\
14.4(0.0-985.6)\end{array}$ & $\begin{array}{l}0.457 \\
0.475 \\
0.193\end{array}$ \\
\hline $\begin{array}{l}\text { Irradiation } \\
\text { technique } \\
\text { 3DCRT } \\
\text { IMRT }\end{array}$ & $\begin{array}{l}81(21.6 \%) \\
294(78.4 \%)\end{array}$ & $\begin{array}{l}82(21.8 \%) \\
294(78.2 \%)\end{array}$ & 0.945 \\
\hline $\begin{array}{l}\text { Chemotherapy } \\
\text { cycle } \\
2 \\
4\end{array}$ & $\begin{array}{l}187(49.9 \%) \\
188(50.1 \%)\end{array}$ & $\begin{array}{l}189(50.3 \%) \\
187(49.7 \%)\end{array}$ & 0.913 \\
\hline
\end{tabular}

Abbreviations: CEA, carcinoembryonic antigen; CA19-9, carbohydrate antigen 19-9; 3DCRT, three-dimensional conformal radiation therapy; IMRT, intensified modulated radiation therapy.

\section{Discussion}

Because of tumor heterogeneity, it is hard to accurately predict RC response to NACRT using only anatomical factors. Hammarström et al reported that tumor length had an AUC of only 0.599 for predicting pCR. ${ }^{17}$ In this study, tumor length and clinical T stage exhibited AUCs of 0.578 and 0.561 for predicting a bad response, respectively. Serum biomarkers are safe and convenient indices which could indirectly reflect dynamic interactions between tumor and host, and may assist in response prediction. Here, we analyzed the association between commonly used serum lipid indices and NACRT response. Based on our results, a pretreatment apoAI of $\leq 1.20 \mathrm{~g} / \mathrm{L}$ led to a higher proportion of bad responders $(60.2 \%$ vs $44.7 \%, P=0.003)$. However, no association was observed between tumor response and TC, TG, HDL-c, LDL-c, or apoB. After excluding the influence of other clinical factors, serum apoAI maintained its prediction independence $(\mathrm{OR}=1.578,95 \% \mathrm{CI} 1.025-2.429)$. To the best of our knowledge, there have been no published clinical studies revealing the impacts of apoAI on chemoradiation sensitivity. Therefore, our study presents apoAI as a new independent predictor of NACRT response in RC.

ApoAI is a subtype of apolipoprotein synthesized by the liver and small intestine. It is known as a cardioprotective protein owing to its roles in reverse transportation of cholesterol from peripheral tissue to the liver. ${ }^{18}$ Its anti-tumor functions have also been demonstrated in recent studies. A series of laboratory studies showed that it could repress tumor growth and metastasis in cell and animal models, through reducing the recruitment of myeloid-derived suppressor cells, increasing the accumulation of $\mathrm{CD} 8^{+}$cytotoxic T-lymphocytes, inducing an M2-to-M1 shift in macrophage phenotype, and inhibiting VEGF-related angiogenesis. ${ }^{19}$ A study by Ben Hassen et al indicated that apoAI could retard the epithelial-to-mesenchymal transition process, which was considered as a key mechanism for chemoradiation resistance of cancer cells. ${ }^{20}$ This provided a possible explanation for the negative correlation between serum apoAI level and tumor response to NACRT.

Similarly, clinical studies showed that the serum level of apoAI correlates positively with prognosis for many solid tumors. Cheng et al reported that a serum apoAI level of $<1.01 \mathrm{~g} / \mathrm{L}$ predicted a poorer overall survival and a higher risk of resistance to platinum-based chemotherapy in non-small cell lung cancer. ${ }^{21}$ Guo et al reported that a serum apoAI level of $<1.04 \mathrm{~g} / \mathrm{L}$ predicted a poorer overall survival in renal cell carcinoma. ${ }^{22}$ A study by Wang et al indicated that low serum apoAI was an independent predictor of shorter overall survival in esophageal carcinoma. ${ }^{23}$ And a study by the present authors indicated that low serum apoAI was an independent predictor of increased distant metastases and poorer overall survival in nasopharyngeal carcinoma. ${ }^{24}$ In colorectal cancer, a decreased level of serum apoAI could also act as an unfavorable prognosticator, for both metastatic and nonmetastatic patients. Moreover, it was found to correlate with reduced efficacy of a VEGF-targeting monoclonal antibody, bevacizumab. ${ }^{15,25,26}$ 


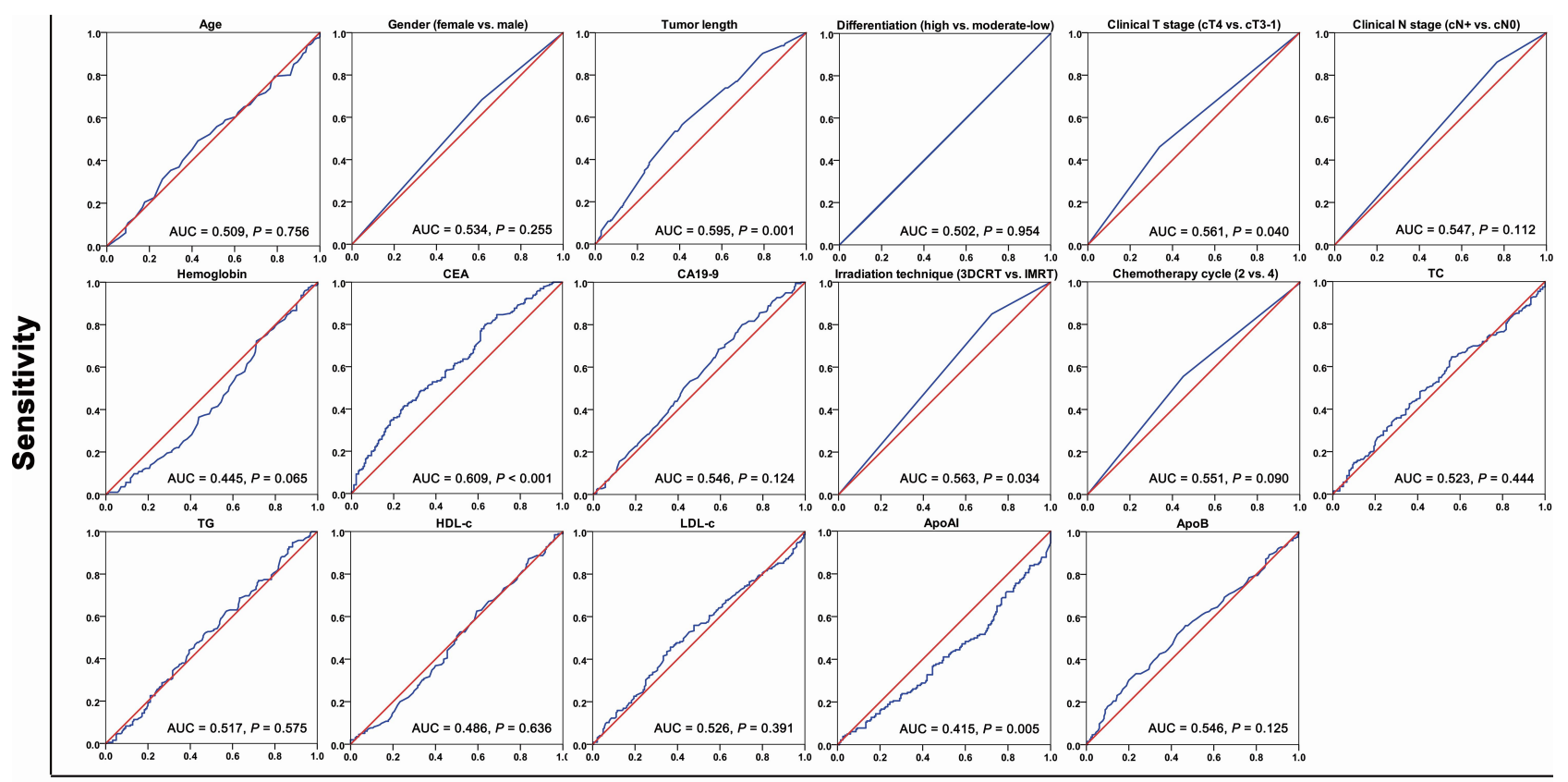

\section{1 - specificity}

Figure 2 Receiver operating characteristics curves of candidate variables for predicting bad response.

Abbreviations: CEA, carcinoembryonic antigen; CAI9-9, carbohydrate antigen 19-9; 3DCRT, three-dimensional conformal radiation therapy; IMRT, intensified modulated radiation therapy; TC, total cholesterol, TG, triglyceride; HDL-c, high-density lipoprotein cholesterol; LDL-c, low-density lipoprotein cholesterol; apoAl, apolipoprotein A-l; apoB, apolipoprotein B; AUC, area under the curve.

In this study, we further developed an easy-to-use PI by combining serum apoAI and four other independent predictors, namely, tumor length, clinical $\mathrm{T}$ stage, serum CEA, and irradiation technique. It appeared to have an
AUC of $0.642(95 \%$ CI $0.586-0.697)$ for predicting a bad response. When a cut-off value of $2(\geq 2$ vs $<2)$ was used, an ideal sensitivity (72.3\%), acceptable positive and negative predictive values $(63.8 \%$ and $61.9 \%$,

Table 2 Chi-Squared Test on Possible Predictors of Bad Response

\begin{tabular}{|c|c|c|c|c|}
\hline Features & Bad Response & Good Response & Chi-Square & $P$ Value \\
\hline \multicolumn{5}{|c|}{ Tumor length $(\mathrm{cm})$} \\
\hline$\geq 4$ & 104 (60.5\%) & 68 (39.5\%) & 9.112 & $0.003 * *$ \\
\hline$<4$ & 91 (44.8\%) & 112 (55.2\%) & & \\
\hline \multicolumn{5}{|c|}{ Clinical T stage } \\
\hline cT4 & $90(59.6 \%)$ & $6 \mathrm{I}(30.4 \%)$ & 5.584 & $0.016 *$ \\
\hline cT3-I & 105 (46.9\%) & $119(53.1 \%)$ & & \\
\hline \multicolumn{5}{|l|}{ CEA $(n g / m L)$} \\
\hline$\geq 5.0$ & I0I (59.4\%) & 69 (40.6\%) & 6.844 & $0.009 * *$ \\
\hline$<5.0$ & 94 (45.9\%) & III (54.I\%) & & \\
\hline \multicolumn{5}{|c|}{ Irradiation technique } \\
\hline 3DCRT & $54(66.7 \%)$ & 27 (33.3\%) & 9.548 & $0.002 * *$ \\
\hline IMRT & I4I (48.0\%) & I53 (52.0\%) & & \\
\hline \multicolumn{5}{|l|}{ ApoAl (g/L) } \\
\hline$\leq 1.20$ & $106(60.2 \%)$ & 70 (39.8\%) & 8.994 & $0.003^{* *}$ \\
\hline$>1.20$ & 89 (44.7\%) & $110(55.3 \%)$ & & \\
\hline
\end{tabular}

Notes: $* P<0.05, * * P<0.01$.

Abbreviations: CEA, carcinoembryonic antigen; 3DCRT, three-dimensional conformal radiation therapy; IMRT, intensified modulated radiation therapy; apoAI, apolipoprotein A-I. 
Table 3 Multivariate Logistic Regression for Predicting Bad Response

\begin{tabular}{|c|c|c|c|c|c|}
\hline Variables & $\beta$ Value & OR & $95 \% \mathrm{Cl}$ & $P$ Value & Points \\
\hline $\begin{array}{l}\text { Tumor length }(\mathrm{cm}) \\
\geq 4 \text { vs }<4\end{array}$ & 0.584 & 1.793 & I.17I-2.747 & $0.007^{* *}$ & I vs 0 \\
\hline $\begin{array}{l}\text { Clinical T stage } \\
\text { cT4 vs cT3-I }\end{array}$ & 0.464 & 1.590 & $1.025-2.465$ & $0.038^{*}$ & I vs 0 \\
\hline $\begin{array}{l}\text { CEA }(\mathrm{ng} / \mathrm{mL}) \\
\geq 5.0 \text { vs }<5.0\end{array}$ & 0.468 & 1.597 & $1.038-2.456$ & $0.033^{*}$ & I vs 0 \\
\hline $\begin{array}{l}\text { Irradiation technique } \\
\text { 3DCRT vs IMRT }\end{array}$ & 0.562 & 1.754 & $1.020-3.016$ & $0.042 *$ & I vs 0 \\
\hline $\begin{array}{l}\text { ApoAl }(\mathrm{g} / \mathrm{L}) \\
\quad \leq 1.20 \mathrm{vs}>1.20\end{array}$ & 0.456 & 1.578 & $1.025-2.429$ & $0.038 *$ & I vs 0 \\
\hline
\end{tabular}

Notes: Each variable was assigned with an integer point nearest to its $\beta$ regression coefficient divided by 0.456 (the smallest $\beta$ value in the model). $* P<0.05$, $* * P<0.01$. Abbreviations: OR, odds ratio; $\mathrm{Cl}$, confidence interval; CEA, carcinoembryonic antigen; 3DCRT, three-dimensional conformal radiation therapy; IMRT, intensified modulated radiation therapy; apoAI, apolipoprotein A-I.

respectively), and an acceptable concordance (63.0\%) were obtained, and it was confirmed that no significant deviation existed between the PI and a perfect fit. Our model was developed from a relatively large cohort in which the mainstream IMRT technique covered $78.4 \%$ of patients. Not only that; it was validated in an independent

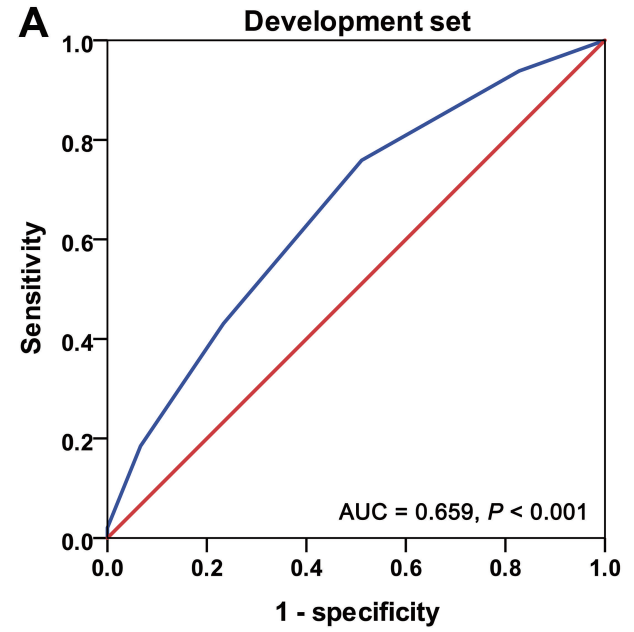

B

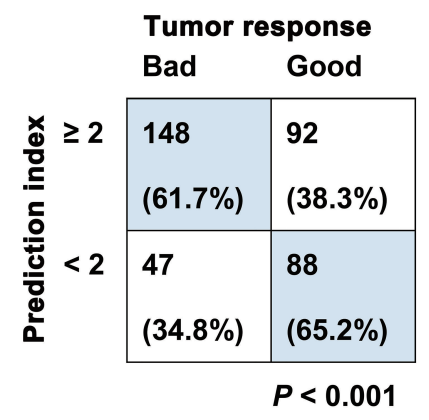

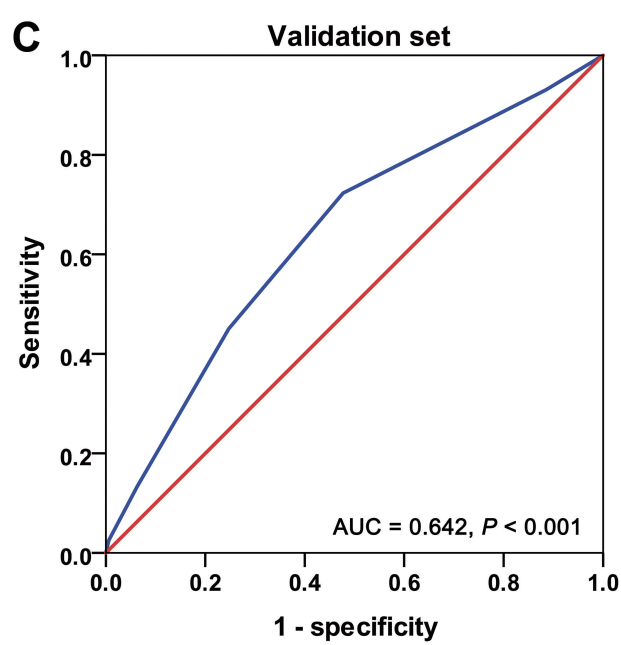

D

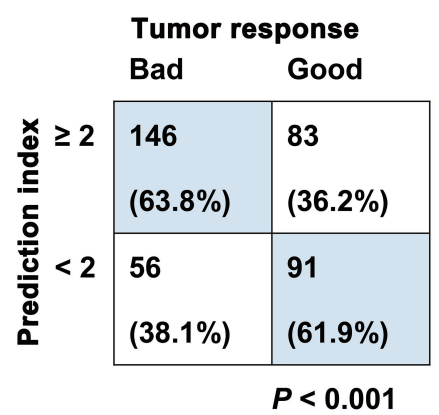

Figure 3 Development and validation of prediction index. (A) Receiver operating characteristics (ROC) curve of prediction index (PI) in the development set; (B) validation of cut-off value for $\mathrm{PI}$ in the development set; (C) ROC curve of PI in the validation set; (D) validation of cut-off value for PI in the validation set. Abbreviation: AUC, area under the curve. 
cohort which also had a relatively large sample size. These advantages made it quite reliable.

Before our study, there have been some prediction models of NACRT response based on serum biomarkers. Sun et al used pre- and post-NACRT levels of serum CEA to build a nomogram for predicting $\mathrm{pCR} .{ }^{27} \mathrm{Hu}$ et al used the CEA clearance pattern to build models for predicting $\mathrm{pCR}$ and TRG. ${ }^{9}$ Tan et al and Engel et al built prediction models for pCR, also by using the pretreatment CEA level. ${ }^{10,28}$ In a model by Song et al, pretreatment CA19-9 level, postNACRT CEA level, pre-post-CEA ratio, and pre-postCA19-9 ratio were used to predict down-staging. ${ }^{11}$ Prediction models, including ours, may assist in making an individualized NACRT plan which balances local control and quality of life. The cases predicted as bad responders may need NACRT with increased intensity, such as a higher dose of irradiation, to improve tumor regression. On the other hand, those predicted as good responders may be eligible for NACRT with reduced intensity, such as a short course of radiotherapy without concurrent chemotherapy, to avoid unnecessary severe toxicity.

In addition, serum apoAI level could be elevated using statin and niacin. ${ }^{29,30}$ Some previous studies reported that NACRT response could be improved using these lipidmodulating agents. In a meta-analysis involving nine clinical trials, statin concurrently with NACRT appeared to increase the pCR rate of RC patients. ${ }^{31}$ Also, in a cytological study, statin exhibited the ability to enhance the radiation sensitivity of RC cells in vitro. ${ }^{32}$ Our results supported apoAI as a new target for NACRT-sensitizing therapies, and added more evidence for using lipid-modulating agents as NACRT sensitizers. On the other hand, apoAI mimetic peptides, including D-4F, L-4F, L-5F, and Tg6F, are now being tried in cancer treatment. These mimetics could be delivered by nanoparticles and transgenic plants, which are safe and well tolerated in humans. A kind of transgenic tomato delivering Tg6F was reported to have promising effects in controlling metastatic colorectal cancer in experimental nude mice. ${ }^{33}$ This may provide another therapeutic selection for improving NACRT response in patients with low levels of serum apoAI.

This study had two main limitations. First, it was a retrospective, single-institutional study without external validation. Its results needed to be further validated by prospective or multi-institutional studies, before clinical generalization. Second, the efficiency of the PI was not good enough and could be improved by involving more patient characteristics, such as radiomic features and new serum biomarkers. But it could still be a simple and practical model suitable for use in hospitals without advanced examination conditions.

\section{Conclusion}

This study found serum apoAI $\leq 1.20 \mathrm{~g} / \mathrm{L}$ to independently predict a bad NACRT response in RC, and built a practical apoAI-based prediction model. These results presented apoAI as a biomarker for guiding individualized treatment, and a potential target for improving chemoradiation sensitivity.

\section{Data Sharing Statement}

The data that support the findings of this study are available from the corresponding author upon reasonable request.

\section{Acknowledgments}

This work was supported by the Guangdong Basic and Applied Basic Research Foundation, China (grant nos 2020A1515010037 and 2020A1515011286). The funding sources had no role in study design, data collection, analysis, interpretation, or manuscript writing. Su-ping Guo and Chen Chen are co-first authors for this study.

\section{Disclosure}

The authors report no conflicts of interest in this work.

\section{References}

1. Petrelli F, Trevisan F, Cabiddu M, et al. Total neoadjuvant therapy in rectal cancer: a systematic review and meta-analysis of treatment outcomes. Ann Surg. 2020;271(3):440-448. doi:10.1097/ SLA.0000000000003471

2. Erlandsson J, Lörinc E, Ahlberg M, et al. Tumour regression after radiotherapy for rectal cancer - results from the randomised stockholm III trial. Radiother Oncol. 2019;135:178-186. doi:10.1016/j. radonc.2019.03.016

3. Fokas E, Ströbel P, Fietkau R, et al; German Rectal Cancer Study Group. Tumor regression grading after preoperative chemoradiotherapy as a prognostic factor and individual-level surrogate for disease-free survival in rectal cancer. J Natl Cancer Inst. 2017;109 (12):djx095. doi:10.1093/jnci/djx095

4. Rijkmans EC, Marijnen CAM, van Triest B, et al. Predictive factors for response and toxicity after brachytherapy for rectal cancer; results from the HERBERT study. Radiother Oncol. 2019;133:176-182. doi:10.1016/j.radonc.2019.01.034

5. Nie K, Shi L, Chen Q, et al. Rectal cancer: assessment of neoadjuvant chemoradiation outcome based on radiomics of multiparametric MRI. Clin Cancer Res. 2016;22(21):5256-5264. doi:10.1158/1078-0432. CCR-15-2997

6. Cho E, Park IJ, Yeom SS, et al. A multigene model for predicting tumor responsiveness after preoperative chemoradiotherapy for rectal cancer. Int $J$ Radiat Oncol Biol Phys. 2019;105(4):834-842. doi:10.1016/j.ijrobp.2019.07.058

7. Akiyoshi T, Tanaka N, Kiyotani K, et al. Immunogenomic profiles associated with response to neoadjuvant chemoradiotherapy in patients with rectal cancer. Br J Surg. 2019;106(10):1381-1392. doi:10.1002/ bjs. 11179 
8. Yang KL, Yang SH, Liang WY, et al. Carcinoembryonic antigen (CEA) level, CEA ratio, and treatment outcome of rectal cancer patients receiving pre-operative chemoradiation and surgery. Radiat Oncol. 2013;8:43. doi:10.1186/1748-717X-8-43

9. Hu H, Huang J, Lan P, et al. CEA clearance pattern as a predictor of tumor response to neoadjuvant treatment in rectal cancer: a post-hoc analysis of FOWARC trial. BMC Cancer. 2018;18(1):1145. doi:10.1186/s12885-018-4997-y

10. Tan Y, Fu D, Li D, et al. Predictors and risk factors of pathologic complete response following neoadjuvant chemoradiotherapy for rectal cancer: a population-based analysis. Front Oncol. 2019;9:497. doi:10.3389/fonc.2019.00497

11. Song J, Huang X, Chen Z, et al. Predictive value of carcinoembryonic antigen and carbohydrate antigen 19-9 related to downstaging to stage 0-I after neoadjuvant chemoradiotherapy in locally advanced rectal cancer. Cancer Manag Res. 2018;10:3101-3108. doi:10.2147/ CMAR.S166417

12. Lee JH, Song C, Kang SB, Lee HS, Lee KW, Kim JS. Predicting pathological complete regression with haematological markers during neoadjuvant chemoradiotherapy for locally advanced rectal cancer. Anticancer Res. 2018;38(12):6905-6910. doi:10.21873/ anticanres. 13067

13. Parhofer KG. New approaches to address dyslipidemia. Curr Opin Lipidol. 2017;28(6):452-457. doi:10.1097/MOL.0000000000000456

14. Agnoli C, Grioni S, Sieri S, et al. Colorectal cancer risk and dyslipidemia: a case-cohort study nested in an Italian multicentre cohort. Cancer Epidemiol. 2014;38(2):144-151. doi:10.1016/j.canep.2014.02.002

15. Quan Q, Huang Y, Chen Q, et al. Impact of serum apolipoprotein A-I on prognosis and bevacizumab efficacy in patients with metastatic colorectal cancer: a propensity score-matched analysis. Transl Oncol. 2017;10(2):288-294. doi:10.1016/j.tranon.2017.01.006

16. Del Boccio P, Perrotti F, Rossi C, et al. Serum lipidomic study reveals potential early biomarkers for predicting response to chemoradiation therapy in advanced rectal cancer: a pilot study. Adv Radiat Oncol. 2017;2(2):118-124. doi:10.1016/j.adro.2016.12.005

17. Hammarström K, Imam I, Mezheyeuski A, et al. Evaluation of associations between routinely collected staging information and the response to (chemo)radiotherapy in rectal cancer. Cancers. 2020;13 (1):16. doi:10.3390/cancers 13010016

18. Lee-Rueckert M, Escola-Gil JC, Kovanen PT. HDL functionality in reverse cholesterol transport-challenges in translating data emerging from mouse models to human disease. Biochim Biophys Acta. 2016;1861(7):566-583. doi:10.1016/j.bbalip.2016.03.004

19. Zamanian-Daryoush M, DiDonato JA. Apolipoprotein A-I and cancer. Front Pharmacol. 2015;6:265. doi:10.3389/fphar.2015.00265

20. Ben Hassen C, Gutierrez-Pajares JL, Guimaraes C, et al. Apolipoprotein-mediated regulation of lipid metabolism induces distinctive effects in different types of breast cancer cells. Breast Cancer Res. 2020;22(1):38. doi:10.1186/s13058-020-01276-9

21. Cheng T, Dai X, Zhou DL, Lv Y, Miao LY. Correlation of apolipoprotein A-I kinetics with survival and response to first-line platinum-based chemotherapy in advanced non-small cell lung cancer. Med Oncol. 2015;32(1):407. doi:10.1007/s12032-014-0407-8
22. Guo S, He X, Chen Q, et al. The effect of preoperative apolipoprotein A-I on the prognosis of surgical renal cell carcinoma: a Retrospective Large Sample Study. Medicine. 2016;95(12):e3147. doi:10.1097/ MD.0000000000003147

23. Wang XP, Li XH, Zhang L, et al. High level of serum apolipoprotein A-I is a favorable prognostic factor for overall survival in esophageal squamous cell carcinoma. BMC Cancer. 2016;16:516. doi:10.1186/ s12885-016-2502-z

24. Chang H, Wei JW, Chen K, et al. Apolipoprotein A-I is a prognosticator of nasopharyngeal carcinoma in the era of intensity-modulated radiotherapy. J Cancer. 2018;9(4):702-710. doi:10.7150/jca. 22836

25. Ye J, Luo QY, Wang XP, et al. Serum apolipoprotein A-I combined with c-reactive protein serves as a novel prognostic stratification system for colorectal cancer. Cancer Manag Res. 2019;11:9265-9276. doi:10.2147/CMAR.S215599

26. Yang DD, Chen ZH, Wang DS, et al. Prognostic value of the serum apolipoprotein B to apolipoprotein A-I ratio in metastatic colorectal cancer patients. J Cancer. 2020;11(5):1063-1074. doi:10.7150/ jca.35659

27. Sun $\mathrm{Y}$, Chi $\mathrm{P}$, Lin $\mathrm{H}$, et al. A nomogram predicting pathological complete response to neoadjuvant chemoradiotherapy for locally advanced rectal cancer: implications for organ preservation strategies. Oncotarget. 2017;8(40):67732-67743. doi:10.18632/ oncotarget.18821

28. Engel RM, Oliva K, Koulis C, Yap R, McMurrick PJ. Predictive factors of complete pathological response in patients with locally advanced rectal cancer. Int $J$ Colorectal Dis. 2020;35 (9):1759-1767. doi:10.1007/s00384-020-03633-8

29. Kakuda H, Matoba M, Nakatoh H, Nagao S, Takekoshi N. Effects of change in high-density lipoprotein cholesterol by statin switching on glucose metabolism and renal function in hypercholesterolemia. J Clin Lipidol. 2015;9(5):709-715. doi:10.1016/j.jacl.2015.07.007

30. Pang J, Chan DC, Hamilton SJ, Tenneti VS, Watts GF, Barrett PH. Effect of niacin on high-density lipoprotein apolipoprotein A-I kinetics in statin-treated patients with type 2 diabetes mellitus. Arterioscler Thromb Vasc Biol. 2014;34(2):427-432. doi:10.1161/ ATVBAHA.113.302019

31. Gash KJ, Chambers AC, Cotton DE, et al. Potentiating the effects of radiotherapy in rectal cancer: the role of aspirin, statins and metformin as adjuncts to therapy. Br J Cancer. 2017;117(2):210-219. doi:10.1038/bjc.2017.175

32. Karagkounis G, DeVecchio J, Ferrandon S, Williams AC, Thomas MG. Simvastatin enhances radiation sensitivity of colorectal cancer cells. Surg Endosc. 2018;32(3):1533-1539. doi:10.1007/ s00464-017-5841-1

33. Delk SC, Chattopadhyay A, Escola-Gil JC, Fogelman AM, Reddy ST. Apolipoprotein mimetics in cancer. Semin Cancer Biol. 2020. doi:10.1016/j.semcancer.2020.11.002
Cancer Management and Research

\section{Publish your work in this journal}

Cancer Management and Research is an international, peer-reviewed open access journal focusing on cancer research and the optimal use of preventative and integrated treatment interventions to achieve improved outcomes, enhanced survival and quality of life for the cancer patient.
The manuscript management system is completely online and includes a very quick and fair peer-review system, which is all easy to use. Visit http://www.dovepress.com/testimonials.php to read real quotes from published authors. 